\title{
1. Thematischer Einstieg
}

Les Syriens on réalisé le plus long film de l'Histoire. Mille et un jours. Les plus longues funérailles de l'Histoire. Cent mille vies et une vie.

Eau Argentèe (2013) (F, R: Mohammed/Bedirxan)

\subsection{Handyvideo - Tod - Syrienkonflikt}

Der Syrienkonflikt begegnet uns - ob als mittelbar Betrachtende ${ }^{1}$ oder direkt und indirekt Betroffene und Beteiligte - seit seinen Anfängen vor allem in Videos: zunächst mit einfachen Handykameras, später zunehmend auch mit hochauflösenden Hand- oder Helmkameras aufgenommen, werden Videos über das Sterben in Syrien über das Internet verbreitet. Von dort aus finden sie Eingang in eine internationale Medienberichterstattung, die sich als objektiv versteht, jedoch selbst in der Hervorbringung eines bestimmten Bildes des Syrienkonflikts eingebunden ist. Solche Videos werden zum Gegenstand von Filmen, Musikstücken und anderen künstlerischen Auseinandersetzungen, die in einer mehr oder weniger offenkundigen Weise ihr eigenes Bild des Syrienkonflikts zeichnen. Den gewaltsamen Tod von Konfliktbetroffenen und -beteiligten zeigend, spielen solche Videos als genuine Dokumente und juristisch verwertbare Beweisstücke, als Propaganda- und Marketingmaterial, als investigative und enthüllende Momente, als cineastisches und spielerisches Erlebnis, als Fake, Pornografie, Obzönität und Horror eine große Rolle für die Wahrnehmung des Syrienkonflikts wie auch für seine Dynamik und öffentliche Resonanz (vgl. Lynch/Freelon/Aday 2014; Meis 2013 u. 2016).

Diese in einem singulären Augenblick aufgenommenen Videos sind dabei zwar als dokumentarische Fragmente eines konkreten Konfliktgeschehens in Syrien ver-

In Hinblick auf eine geschlechtersensible Schreibweise werden in diesem Buch geschlechtsneutrale Funktionsbezeichnungen verwendet, die sowohl die weibliche als auch die männliche Form einschließen. Ist dies aufgrund sprachlicher Restriktionen nicht möglich, ist mit der Nennung der männlichen Funktionsbezeichnung, sofern nicht anders gekennzeichnet, immer auch die weibliche Form gemeint. 
ortet, sie weisen aber auch stets darüber hinaus. So bspw. auf die Grüne Bewegung als eine junge Studentin namens Neda auf den Straßen Teherans am 20. Juni 2009 durch den Schuss eines (vermeintlich) regimetreuen Milizionärs während einer friedlichen Demonstration zu Tode kam. Ein Video von ihrem Sterben in niedriger Handykameraqualität wurde noch am gleichen Abend auf YouTube hochgeladen und ging binnen kürzester Zeit viral. ${ }^{2}$ Unmittelbar nach seiner Veröffentlichung wurde dieses Video auch auf anderen Social-Media-Plattformen wie Twitter und Facebook in einer grünen Welle der kollektiven Trauer und internationalen Solidarität verlinkt, kommentiert und weiterverbreitet. Gleich darauf wurde Nedas Tod in internationalen Nachrichtensendungen betrauert und verurteilt. Sie wurde zur Ikone und zum Symbol des zivilen Widerstands gegen ein brutales Regime. Ihr Gesicht wurde zum Aushängeschild der Grünen Bewegung, deren Antrieb zunächst der Protest gegen die (vermeintlich) gefälschte iranische Präsidentschaftswahl war. Nicht zuletzt vor dem Hintergrund von Nedas Tod wurde diese jedoch zu einer Bewegung für soziale Gerechtigkeit und Freiheit. In den folgenden Monaten wurden Neda, ihr Leben und ihr Tod zum Gegenstand zahlreicher Dokumentationen und international geführter Debatten, die ein uneindeutiges Bild ihres Ablebens zeichneten: Auf der einen Seite der Mord an einer jungen Studentin für den Machterhalt eines despotischen Regimes. Auf der anderen Seite ihre willentliche Opferung für die Ziele oppositioneller Kräfte im Iran und das Resultat einer internationalen Verschwörung gegen den Iran (vgl. Bashi 2010: 40; Sabety 2010: 121; Bach Malek 2010: 287; Dehghan 2010; Emamzadeh 2011: 24; Meis 2014: 85ff.).

Nedas Tod wurde so zu einer Geschichte, die in Gedichten, Liedern, Filmen, Musikvideos und anderen Videowerken geschrieben und auf sozialen Plattformen wie YouTube stetig aktualisiert wird. ${ }^{3}$ Es ist eine Geschichte mit gegenläufigen Erzählsträngen, die bspw. in kriminalistisch investigativen ${ }^{4}$ Videos auf YouTube sichtbar werden und sich im Gegenüber einer westlichen Deutung der Geschehnisse um Nedas Tod positionieren. Auch ist es eine Geschichte überraschender Wendungen: So kam es, dass sich kurz nach Öffentlichwerden von Nedas Tod die Für-

2 Im Frühjahr 2019 wurde das Video alleine auf dem YouTube-Kanal FEELTHELIGHT 2.378.342mal aufgerufen. Siehe: <https://www.youtube.com/watch?v=bbdEfoQRsLM>.

Siehe hierzu bspw. Songs, die in Reaktion auf Nedas Tod geschrieben wurden: I Am Kawehi 2013 <https://www.youtube.com/watch?v=k66S471]3as>, Kiddam And The People $2016<$ https ://www.youtube.com/watch?v=3mNEgLWicN4> oder squarerootofjess $2016<$ https://www.yo utube.com/watch?v=htvGYi93CD8>. von Neda inszeniert wurde (91177info $2010<$ https://www.youtube.com/watch?v=E18bWaQLAo\&t=11s\&bpctr=1544172309>) oder die den Fake-Charakter des Handyvideos von ihrem Tod in einer analytischen Beweisführung zu belegen suchen (detectivemoonk $2009<\mathrm{https}$ ://ww w.youtube.com/watch?v=CByrgILwGaM\&bpctr=1530607563>). 
tot-Erklärte auf einmal in sozialen Medien $^{5}$ und internationalen Nachrichtenmedien zu Wort meldete. Denn in der Anfangsphase der Berichterstattung wurde Neda Soltani fälschlicherweise als die Frau im Video auf Grundlage der Meldungen und Bilder auf Twitter und Facebook identifiziert, deren Nachname große Ähnlichkeit mit dem der später für tot erklärten Neda Agha-Soltan aufweist. Der Name und das Foto von Neda Soltani wurden in internationalen Nachrichtensendungen veröffentlicht. Wenig später war ihr Gesicht nicht nur weltweit in Zeitungen zu sehen, sondern auch auf den Protestplakaten der Grünen Bewegung. Für die lebende Neda Soltani hatte diese Verwechslung weitreichende Konsequenzen: Sie wurde von der iranischen Regierung unter Druck gesetzt und musste aus dem Iran fliehen, nachdem die Versuche, ihr >gestohlenes` Gesicht zurückzugewinnen, scheiterten (vgl. Schraven 2010; Emamzadeh 2011: 24).

Dieser Vorfall und seine Konsequenzen liegen dabei nicht allein in der übereilten Reaktion von Journalisten »in Zeiten der Hysterie« (Schraven 2010) begründet. Ebenso ist er der speziellen Ästhetik des Handyvideos von Nedas Tod und der technologischen Bedingungen der sogenannten sozialen Medien geschuldet: Die 37 Sekunden des Videos sind verwackelt und unscharf. Das Gesicht der jungen Iranerin, die gestützt von zwei Männern zu Boden geht, ist zunächst kaum im Video zu sehen. Als die Frau am Boden liegt geht die Kamera zwar näher heran, jedoch lassen der Aufnahmewinkel sowie die Instabilität und Unschärfe des Bildes das Gesicht kaum klar erkennen, bevor es von Blut überströmt ist und weitere Passanten in der chaotischen Szene immer wieder die Sicht der Kamera versperren. Auch die Audioqualität ist undeutlich: die Stimmen sind dumpf und überlagert von Hintergrundgeräuschen und Schreien (vgl. FEELTHELIGHT 2009). Auf Grundlage dieses Ausgangsmaterials, des im Video fallenden Vornamens Neda und der unvollständigen Informationen in den sozialen Medien zum Nachnamen und Hintergrund der Frau im Video, identifizierten Journalisten über Facebook Neda Soltani, kopierten

Der Begriff soziale Medien wird hier mit Rückgriff auf van Dijck (2013: 11f.) für soziotechnische Onlineplattformen verwendet, die auf der einen Seite nutzerzentriert sind, zwischenmenschliche Netzwerke unterstützen und im Sinn einer Begünstigung sozialer Verbundenheit den sozialen Zusammenhalt erweitern. Auf der anderen Seite werden hierunter automatisierte Systeme einer technisch entworfenen und arrangierten Konnektivität begriffen, die auf der Kodierung von Wünschen, Vorlieben und Vorstellungen der Nutzenden und deren Übersetzung in Algorithmen basiert. Foto- und Videoinhalte sind in der dafür notwendigen automatisierten Datensammlung zu den sozialen Beziehungen der Nutzenden ein entscheidender Faktor. Diese Datensammlung wird angetrieben von Fragen wie: Wer teilt welche Bilder mit wem? Welche Bilder oder Videos sind bei welchen sozialen Cruppen beliebt? Wer sind die stastemaker in diesen Cemeinschaften? Entsprechend dieser soziotechnischen Verfasstheit verweist van Dijck auch darauf, dass der Begriff »connective media« (ebd.: 13) gegenüber Social Media zu bevorzugen sei. Da jedoch die Begrifflichkeiten Social Media bzw. soziale Medien den allgemeinen Sprachgebrauch im nicht-wissenschaftlichen wie auch im wissenschaftlichen Bereich prägen, werden in dieser Arbeit auch ebendiese verwendet. 
ihr Facebook-Profilbild und verbreiteten es über verschiedene Medienkanäle weiter (vgl. Schraven 2010).

Im Blick dieser tragischen Verwechslung erhalten nicht nur die Deutungsversuche des iranischen Regimes, die von der Inszeniertheit des Videos bis zu einer Vereinnahmung und zum Komplott westlicher Medien reichen (vgl. ebd.), Substanz. Auch zeigt sich die Bedeutsamkeit medienästhetischer Charakteristika, die geradezu typisch für >Low-tech-Videoaufnahmen sind wie sie 2009 im Iran Verbreitung fanden. Die Bezeichnung >low-tech rekurriert hier auf Fiskes (2002: 387) Gegenüberstellung von >videolow< und `videohigh`, die er mit entsprechenden sozialen Bereichen in Verbindung bringt. Auf der einen Seite sieht er den Bereich des geringen Kapitals, der geringen technologischen Verfügbarkeit und Ausstattung und der geringen sozialen Macht. Auf der anderen Seite verortet er den Bereich des hohen Kapitals, der gehobenen Technologie und der hohen sozialen Macht mit einer entsprechend gesteigerten Möglichkeit zur technologischen und sozialen Intervention. Als ästhetische Merkmale des >videolow< führt Fiske dabei »poor but closely involved vantage points, moments of loss of technical control (blurred focus, too-rapid pans, tilted or dropped cameras), and [...] reduced editing« (ebd.:389) an. Gerade diese ästhetischen Merkmale sind es, die auch >Low-tech $<-V i d e o a u f n a h m e n$ im Syrienkonflikt auszeichnen und die hinsichtlich ihrer öffentlichen Wahrnehmung durch eine starke Ambivalenz geprägt sind: Auf der einen Seite stehen gerade amateurhafte Handyaufnahmen von direkt Betroffenen und Beteiligten aus der Mitte eines Konflikts für eine unverfälschte, unmittelbare und unbearbeitete Genuität, die sie zu einem besonders glaubhaften Dokument im Konfliktkontext machen. Vor allem dieser Aspekt ist es, der im Rahmen der iranischen Grünen Bewegung internationale Solidarität hervorbrachte und das Handy zum Widerstandssymbol werden ließ (vgl. Meis 2014: 79ff.). Auf der anderen Seite zeichnen sich solche >Low-tech--Handyvideos durch eine medienspezifische Offenheit für diskursive Zuschreibungen und Deutungen, für Reproduktionen und Modifikationen aus, die sich aus der geringen Aufnahmequalität ${ }^{6}$ und dem Möglichkeitsraum digitaler Bild- und Videotechnologien und des Internets ergibt. Dieser Möglichkeitsraum bringt zudem eine hohe Veränderlichkeit und modale Zweifelhaftigkeit mit sich (vgl. Hüppauf 2008: 560ff.; Krenzer 2013: 29ff.; Meis 2013).

Von ästhetischen wie auch diskursiven Gesichtspunkten aus betrachtet zeichnen sich diese Videos somit durch eine Uneindeutigkeit aus, die sie als oszillierende Ereignisse zwischen Leben und Tod, zwischen Fake ${ }^{7}$ und Authentizität, zwischen

Typisch für eine geringe Aufnahmequalität sind u.a. verpixelte, verwackelte und unscharfe Bilder, unruhige und zu schnelle Schwenks, schräge oder abgeschnittene Einstellungen oder ein unklarer und chaotischer Ton.

7 Wie Martin Doll (2012: 24f.) in seiner diskurskritischen Arbeit zu Akten der Täuschung festhält, ist der Begriff des Fakes zwar eng mit dem Begriff der Fälschung verwandt, aber je nach 
Obszönem und Angemessenem niemals stillstehen lässt. Weder lassen sich einzelne Videos auf einen dieser Pole fixieren noch lassen sie sich in ihrer Bedeutung festschreiben, weil diese sowohl von dem Erscheinungs- und Betrachtungskontext als auch von einer spezifischen Temporalität und Lokalität der Videos abhängig ist. Denn wie es Malte Hagener (2011: 47) für den Film beschreibt, sind auch diese Videos in der Umgebung digitaler Medienkonvergenz durch eine mediale, räumliche und zeitliche Instabilität bzw. Veränderlichkeit und Dispersität gekennzeichnet, die verschiedenste Assoziationen, Interpretationen, Relationen und Reproduktionen zulässt. Im Fall von Handyvideos drückt sich dieser Umstand vor allem in ihrer Flüchtigkeit und Situiertheit aus. Zusammen mit der eben beschriebenen Uneindeutigkeit werden je nach Einbettung der Videos in die verschiedensten ästhetischen und diskursiven Zusammenhänge ganz unterschiedliche Assoziationen und Verbindungen $\mathrm{zu}$ anderen Videoereignissen und weiteren medialen Materialisierungen, zu gegenwärtigen und vergangenen Konflikten, zu soziopolitischen Debatten und Wissensfeldern aufgerufen, die die Bedeutung der Videos - mehr oder weniger dominant - (mit-)bedingen. Neben den verschiedensten Diskursbezügen und ästhetischen Parallelen zwischen unterschiedlichen Konflikten, wie sie sich für den Iran und Syrien, aber auch allgemein für andere Konflikte des Arabischen Frühlings ${ }^{8}$ (insb. Ägypten oder Libyen) zeigen, sind hier auch motivgeschichtliche und ikonografische Bezüge relevant. Diese stützen sich auf ein kollektives Bildgedächtnis, das häufig unter den Vorzeichen >christlich` und >islamisch` religiös-

Sprachgebrauch und Kontext nicht unbedingt sinngleich mit diesem zu sehen. Eine begriffliche Divergenz liegt bspw. dann vor, wenn der Fake auf eine aufdeckende Ceste verweist, die in einem betreffenden Artefakt oder Phänomen genuin mit angelegt ist. Zur selben Zeit findet der Begriff des Fakes jedoch auch gleichbedeutend mit dem Begriff der Fälschung Verwendung im Sinn einer Person, eines Artefakts oder Phänomens, das vorgibt etwas zu sein, was es nicht ist. In der Betrachtung von Handyvideos in dieser Arbeit begegnen sich beide Bedeutungen des Fake-Begriffs in unterschiedlichen Zusammenhängen und Verstrickungen, wenn Videos im Vorhinein mit einer overten Motivation des Fakens produziert und veröffentlicht werden, oder im Nachhinein durch eine Community von Nutzenden sozialer Medien und/oder (selbst-)ausgewiesenen Experten als Fake benannt werden.

8 Mit dem Arabischen Frühling wird auf eine größere Protestwelle in der MENA-Region (>Middle East \& North Africaく; Ländergürtel von Marokko bis Iran) verwiesen, die Mitte Dezember 2010 ihren Anfang in Tunesien nahm als Straßenverkäufer Mohamed Bouazizi sich selbst anzündete, weil er keine Lebensperspektive mehr für sich sah. Denen in Tunesien folgten Proteste in Ägypten, Marokko, dem Jemen, dem Oman, Libyen, Syrien und Jordanien. Viele andere arabische Länder folgten in den kommenden Monaten. Auch in nicht-arabischen Ländern wie China und dem Iran fanden Demonstrationen statt, die mit dem Arabischen Frühling in Verbindung gebracht werden. (vgl. Asseburg 2011: 7; Rosiny/Richter 2016) Mitte 2017 wurde kurzzeitig das Aufkeimen eines zweiten Arabischen Frühlings konstatiert als es in Marokko zu erneuten Protesten gegen Arbeitslosigkeit und strukturelle Benachteiligung kam (vgl. Ehlert 2017). 
kulturell fundiert ist. Je nach Betrachtungskontext und je nach Betrachtungsperspektive nehmen diese Videos so eine andere Bedeutung ein. Mit anderen Worten: Je nach Situierung der Betrachtung bedeuten die Videos den mit ihnen im $\mathrm{Zu}$ sammenhang stehenden Konflikt, den Tod und die mit ihnen in Zusammenhang stehenden Medientechnologien (einschließlich ihres technischen Apparats und ihrer soziopolitischen und soziokulturellen Praxis) in einer anderen Weise. Damit können diese Videos als Kristallisationspunkte situierten Wissens im Sinn Donna Haraways (1988: 592f.) verstanden werden, da sie als Wissensobjekte selbst eine bedeutungsgenerierende Kraft sind und die Darstellungen der >wirklichen Welt der Logik einer machtgeladenen sozialen Konversationsbeziehung mit ihnen folgt.

Die angesprochene ästhetische Uneindeutigkeit und diskursive Unabgeschlossenheit der Videos erscheinen in diesem Zusammenhang umso gewichtiger, als dass sich ihr primärer Gegenstand - der Tod - durch ganz ähnliche Merkmale in Bezug auf seine wesenseigene Unfassbarkeit (vgl. Macho/Marek 2007) auszeichnet. Die >unscharfeく Gegenwart des Todes scheint sich in der >Low-tech-Ästhetik und diskursiven Offenheit der Videos zu wiederholen. Hierbei lassen diese Videos die Betrachtenden nicht unbeteiligt, sondern binden sie aufgrund ihres Todesbezugs sowie ihrer medienästhetisch und -technologisch bedingten Unmittelbarkeit in den Moment ihrer Bedeutungsgenerierung ein. Beispielhaft zeigt sich dies an einem Handyvideo, das erstmals auf dem YouTube-Kanal netspanner unter dem Titel Man films his own death in syria protest veröffentlicht wurde. Das betreffende Video wurde dabei auf einem YouTube-Kanal veröffentlicht und kommentiert, der keine direkte Verbindung zum syrischen Medienaktivismus oder irgendeiner spezifischen Konfliktpartei aufweist. ${ }^{9}$ Damit rückt das Video in das Bedeutungsspektrum eines besonders authentischen Dokuments eines sensationellen Ereignisses (vgl. netspanner 2011). Neben verschiedenen Nachrichtenkanälen (wie al-Jazeera English, Spiegel Online und The Guardian), die das Video in den Kontext eines profes-

Das Video ist unter <https://www.youtube.com/watch?v=j5]PFHL5rCk\&bpctr=1544175210>abrufbar. Auf dem Kanal netspanner stehen im Frühjahr 2019 insgesamt 30 Videos zur Verfügung, die ungefähr im Zeitraum von 2011 bis 2012 hochgeladen wurden und sich ganz unterschiedlichen Kategorien zuordnen lassen: lustige Tiere (Wormcat is watching), Missgeschicke und peinliche Situationen (Firework goes wrong funny), Naturphänomene und -katastrophen (Giant waves at sea), Kurioses (Tree climbing robot), Unfälle (Truck trailer hits overhead sign), einmalige Bauwerke (World's Longest Over-Water Bridge), historische Szenen (First contact with the Touloumbis tribe in 1976) sowie Gewalt- und Konfliktszenen (Dutch boarding team ambushed by Somali pirates, firefight). Aufgrund dieser Sammlung an unterschiedlichen Videos, die vor allem spektakuläre Ereignisse und Außergewöhnliches betreffen, kann davon ausgegangen werden, dass dieser Kanal keine Verbindung zum syrischen Medienaktivismus oder einer syrischen Konfliktpartei unterhält. Hierfür spricht auch, dass keine weiteren Kanäle empfohlen werden und keine zusätzlichen Kanalinfos oder Diskussionen auf der Seite des Kanals vorhanden sind. Somit ist anzunehmen, dass das Video nicht vor dem Hintergrund etwaiger Konfliktinteressen auf YouTube hochgeladen wurde. 
sionellen Journalismus setzen, der im Gegenüber eines amateurhaften Bürgerjournalismus angesiedelt ist (vgl. al-Jazeera English 2011; Black/Hassan 2011; Schröder 2011), ${ }^{10}$ griff auch der libanesische Künstler Rabih Mroué dieses Handyvideo auf. Mroué stellt das Video innerhalb seiner >non-academic Lecture/Performance The Pixelated Revolution ${ }^{11}$ (2012) dabei in Bedeutungszusammenhänge, die auf verschiedenste Weise das Verhältnis von Leben und Tod im Syrienkonflikt thematisieren (vgl. WRO Art Center 2013).

Dieses Video, das von Mroué »double shooting« (ebd.) genannt wird, zeigt wie ein syrischer Handyfilmender seinen Todesschützen filmt. In der auf YouTube verfügbaren Version zieht das Video die Betrachtenden abrupt in eine aufwühlende Szene hinein, die mit den verwackelten und unscharfen Bildern von Wohnkomplexfragmenten beginnt, die auch in Mroués Präsentation zu sehen sind. Im Hintergrund sind gleich zu Beginn Schüsse, entfernte Rufe, Schreie und eine aufgeregte arabisch-sprechende Männerstimme zu hören (vgl. netspanner 2011: min. 0:00०:09), die, wie man aus Mroués Präsentation erfährt, die Situation kommentiert. Mroués Übersetzung hierzu ins Englische lautet: »The security forces are shooting at our fellow citizens on Sham street, in the neighborhood of Karam al Shami on July 1 2011, for no apparent reason. ${ }^{12}$ There is no demonstration or anything like that.« (WRO Art Center 2013: min. 00:45-01:31) Die Aufregung ist deutlich in der Stimme des Kameramannes zu hören. Er atmet schwer. In direkter Nähe zur Kamera ist mindestens eine weitere Männerstimme zu vernehmen. Etwas, das aussieht wie die gemauerte Brüstung eines Balkons, von dem aus der Kameramann filmt, kommt einen Moment von unten ins Bild. Der Kameramann scheint auf die andere Seite des Balkons zu laufen, denn das Bild wird für einen kurzen Moment noch unruhiger. Im Hintergrund ertönen ein Pfeifen und Kreischen. Dann ist ein tet.

Dieser journalistische Bedeutungszusammenhang wird eingehender in Kapitel 3.2 betrach-

Ein Ausschnitt der Videoversion von Mroués >Lecture/Performance< ist unter <https://vimeo. com/119433287> abrufbar.

Bereits in dieser Aussage im Video tritt die besondere Situiertheit der Handy-Todesvideos hervor. Diese erscheint als eine stark kontext- und betrachtungsabhängige Situationswahrnehmung, die das Handyvideo in einer sehr bestimmten Weise bedeutet. Denn vor dem Hintergrund der historischen, religiösen und soziopolitischen Gegebenheiten in Syrien ließen sich durchaus Gründe (ob ethisch bzw. moralisch vertretbar oder nicht, sei dabei außen vorgelassen) für diese Übergriffe nennen: In Homs leben überwiegend Sunniten, weshalb die Stadt einen potentiellen Gefahrenherd aus Sicht der alawitischen Regierung unter Bashar al-Assad darstellt. Gegenströmungen in sunnitisch dominierten Regionen werden von ihr als existentielle Bedrohung aufgefasst und häufig mit militärischer Gewalt beantwortet. Ein historisches Beispiel für ein solches Vorgehen ist das sogenannte Massaker von Hama im Jahr 1982 als die sunnitische Opposition der Muslimbruderschaft gewaltsam und unter Inkaufnahme zahlreicher ziviler Opfer von dem syrischen Regime niedergeschlagen wurde. (vgl. Schweizer 2016) 
Teil eines anderen Hochhauses zu erkennen, an dessen Fuß sich der Todesschütze - von dem zunächst nur die Stiefel, dann die Beine sichtbar sind - vom linken oberen Bildrand langsam, dicht entlang der Gebäudemauer, der Kamera nähert. Das Bild bleibt unruhig, reißt immer wieder ab, zeigt Fragmente des Himmels, des Gebäudes und der Straße mit parkenden Autos. (vgl. netspanner 2011: min. 0:10-0:41; Abb. 1)

Abb. 1: netspanner (2011): Man films his own death in Syria protest

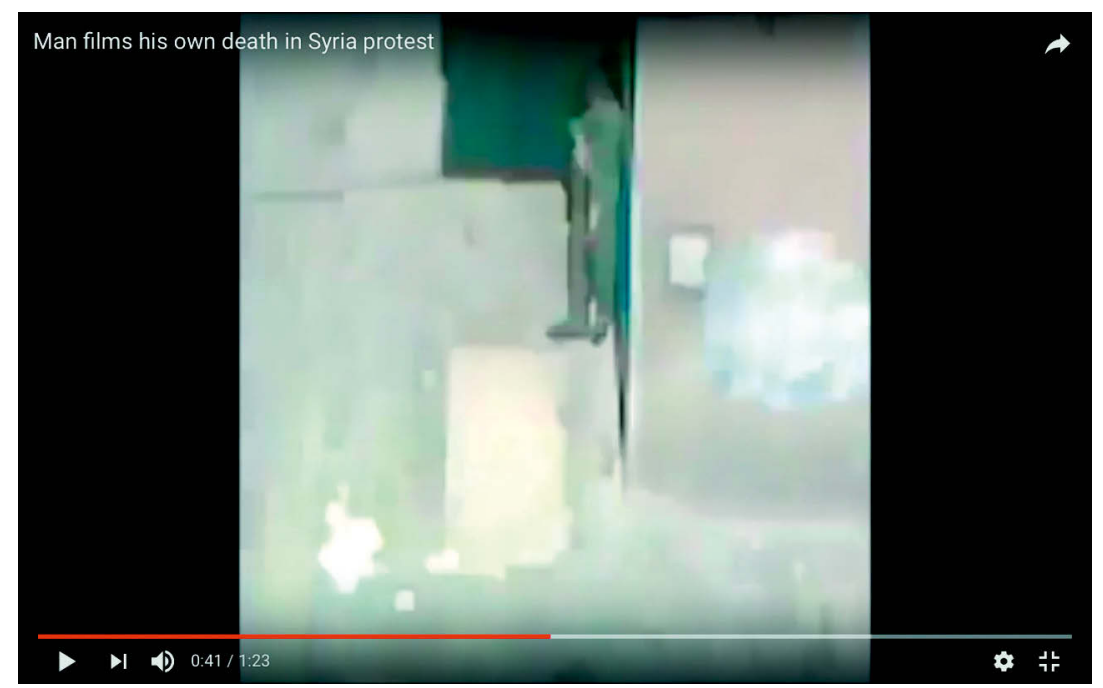

An dieser Szene setzt Mroués Performance an (vgl. WRO Art Center 2013). Die folgenden Bilder fangen wieder den sich nähernden Todesschützen ein. Er hat sich bis zur Ecke des Gebäudes vorgearbeitet und erscheint nun in voller Größe im Bildausschnitt: Bekleidet mit einem grünen Springeranzug hält er ein Gewehr in Bereitschaftsposition. Er bringt das Gewehr in Anschlag. Das Bild bleibt verwackelt. Es verliert kurz den Mann mit dem Gewehr und zeigt ein weiteres Fragment des Wohngebäudes, während im Hintergrund aufgeregte Rufe und das Hantieren mit dem Handy zu hören sind. Das Bild findet den Mann in schussbereiter Position wieder. Ein alles übertönender Schuss erschallt und die Aufnahme fällt völlig aus dem Rahmen: Ein helles, fast grelles Bild gefolgt von einem rotkarierten Bild und begleitet von knackenden, dumpfen Tönen, als würde etwas auf den Boden fallen, sind zu sehen. Dann folgen wieder helle, zunächst weiße, danach gelbliche Bilder und schließlich weißgrauer Stillstand. Auch auf der Tonebene herrscht einige Sekunden lang Stille bis eine wimmernd murmelnde Männerstimme und eine weitere, aufgeregt arabischsprechende Männerstimme lauterwerdend ertönen. Nach 
wenigen Sekunden folgen schreiende Ausrufe einer weiteren Männerstimme. Erneut hallen Schüsse im Hintergrund. (vgl. netspanner 2011: min. 0:42-1:02) Mroué interpretiert diese >leeren « Bilder als die Zimmerdecke, die die zu Boden gefallene und aufwärts gerichtete Kamera aufzeichnet. Die wimmernde Stimme benennt er als die des Kameramannes und übersetzt: "I am wounded. I am wounded. I am wounded.« (WRO Art Center 2013: min. 02:22-02:38) Im YouTube-Video reißt der Ton noch einmal ab. Dann sind für knapp 20 Sekunden immer wieder Männerstimmen zu hören, die miteinander reden, während weiterhin Schüsse im Hintergrund ertönen. Nach einer Minute und 23 Sekunden endet das Video unvermittelt. (vgl.

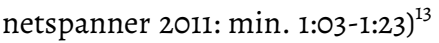

Laut den Informationen auf YouTube wurde das Video am 04.07.2011 hochgeladen. Dies bestätigt eine Meta-Daten-Extraktion. ${ }^{14}$ Auch ist eine kurze englische Beschreibung des Videos verfügbar: "Man films his own death while covering a protest in Syria« (netspanner 2011) sowie eine unvollständige Übersetzung des gesprochenen Arabischen ins Englische:

»0:00 - the armed services are shooting at my country men for no reason on $1 / 7 / 2011$, there is no protest or anything.

0:13 - Background noises from other people, I couldn't really make it out.

$0: 41$ - the cameraman is shot.

0:54 - guy \#2 says: > the bullet entered your head?!?!

0:56- guy \#2 says: > Oh God, Oh God!!!!

1:03 - I couldn't make out the victim's mumbling.

1:11 - guy \#2 says: > what, you were filming?!?!« (ebd.)

Im Licht dieser Übersetzung erscheint das Handyvideo mit seinen verwackelten Szenen von Wohngebäudefragmenten und ausschnitthaften Bildern des Schützen als unintendierte, akzidentielle Sequenz, die aus der lebensbedrohlichen Situation resultiert, in der sich der Filmende befindet und die seine Aufregung und Angespanntheit widerspiegelt. In The Pixelated Revolution werden die gleichen Szenen zu einer aktiven Handlung umgedeutet: die Suche des Kamerauges/-mannes ${ }^{15}$ nach dem Schützen. Insgesamt analysiert und synthetisiert Mroué dieses YouTubeVideo zu einer im Vorlesungsstil gehaltenen Präsentation, die die Nutzung von Handykameras und -videos durch syrische Demonstrierenden als eine gewaltlose

13 Eine ausführliche Beschreibung des Videos findet sich im Anhang unter 6.1.

14 Mithilfe des YouTube-Dataviewers von Amnesty International lassen sich Datum und Zeit des Uploads sowie die Video-ID extrahieren. Gleichzeitig wird eine Rückwärtsbildsuche durchgeführt mit der anhand der Videobildvorschau (engl. sthumbnailı) ermittelt werden kann, ob ein Video schon einmal oder mehrmals im Internet veröffentlicht wurde. Siehe: <www.a mnestyusa.org/citizenevidence/>.

15 Dieses vereinigende Moment von Kamera-Auge-Kameramann wird in Kapitel 3.1 wieder aufgegriffen. 
Waffe des Protests thematisiert. Hierbei werden nicht nur Parallelen zu den visuellen Phänomenen der Aufstände des Arabischen Frühlings, der cineastischen Ästhetik und dem Verhältnis von Bild und Tod gezogen (vgl. Fasshauer 2012; Wilson-Goldie 2012; Mroué 2012; WRO Art Center 2013). Auch beschreibt Mroué das Video in einer sehr bestimmten Weise: ")One person is shooting with a camera, the other is shooting with a rifle. One shoots for his life and the life of his citizens. And the other is shooting for his life and the life of his regime. « (WRO Art Center 2013: min. 00:29-00:42) In der Ungewissheit über das Schicksal des Filmenden, der um sein Leben und das Leben der Syrer filmt, wird das Video zu einem Moment stilisiert, an dem der Tod unbestimmt bzw. ausgesetzt ist. Die Handyvideos werden bzw. das Filmen mit der Handykamera wird nicht bloß zu einem ästhetischen Kampfwerkzeug, sondern zu einer Überlebensstrategie. Von dieser Perspektive aus betrachtet stellen die syrischen Protestierenden mit den Handyvideos über ihren Tod den letzten Augenblick ihres Lebens der tödlichen Militärgewalt des syrischen Regimes entgegen. ${ }^{16}$

Auf der dOCUMENTA (13) 2012 in Kassel präsentierte Mroué auch eine mehrteilige Installation, die seine >Lecture/Performance ergänzt. Zum einen besteht diese aus einem Wandtext von Empfehlungen und pragmatischen Anweisungen zum Filmen von Protesten und Demonstrationen, die sich an das kinoästhetische Manifest Dogma $95^{17}$ anlehnen. Zum anderen enthält die Installation mehrere Übersetzungen des digitalen Ausgangsmaterials in analoge Medien, die die medienästhetischen Charakteristika desselben nur umso deutlicher ausstellen, desto vehementer sie sie zu neutralisieren suchen: Postergroße Ausdrucke der verpixelten Gesichter der Täter aus den YouTube-Videos, die in der >Lecture/Performance`zitiert werden, sollen der Identifikation dieser dienen. Dies scheitert jedoch an der schlechten Qualität und Flüchtigkeit des Videobildes und stellt lediglich eine gesichtslose kollektive Identität des syrischen Regimes aus. ${ }^{18}$ Die endlose Projektion einer

16 Dieser Aspekt wird in Kapitel 3 wieder aufgegriffen und weiter ausgeführt.

17 Von den dänischen Regisseuren Lars von Trier, Thomas Vinterberg, Kristian Levring und Soeren Kragh-Jacobsen mit einer Flugblattaktion »auf einem internationalen Symposium anlässlich des 100. Geburtstages des Films im Pariser Odeon-Theater (Maneljuk 2007) ins Leben gerufen, stellt das Manifest >die Zehn Gebote` eines kinematografischen Fundamentalismus auf, die den Film von den Genrekonventionen und der Hervorsagbarkeit des Hollywoodkinos befreien sollte.

18 Das Sezieren eines Videos in seine einzelnen Standbilder mit einer aufklärerisch ermittelnden Intention fand bereits im Rechtsfall Rodney King Anwendung. Auch damals war dieses Vorgehen wenig zielführend, da die in der Beweisführung vor Gericht analysierten Standbilder wenig zur Rekonstruktion des Tathergangs und zur Urteilsfindung beitrugen, wenn nicht gar diese behinderten. Ronell (2000: 257f.) führt dies darauf zurück, dass die Zerstückelung des Videos in seine Standbilder und die Einpassung dieser in den Rahmen einer Cerichtsverhandlung nichts weiter zum Vorschein brachten als ein von xenophobischen Vorstellungen und rassistischen Stereotypen überschriebenem Phantombild, dessen inhärente 
Schattenfigur mit Handykamera vor rotem Hintergrund, die erschossen zu Boden fällt und sich im Rückwärtsabspielen der Szene wieder aufrichtet, um gleich darauf durch denselben Schuss erneut zu Boden zu fallen, kann als Versuch gesehen werden, den Moment des Todes in seiner Zeitlosigkeit und Flüchtigkeit, welche in den Handyvideos medienästhetisch verdoppelt wird, zu bändigen. Sichtbar wird stattdessen nicht mehr als der Effekt: Der Lebende und der Tote. Sieben Daumenkinos mit den ausgedruckten Einzelbildern von YouTube-Videos befestigt auf einem Tisch mit jeweils darüber installierten Lautsprechern, die auf Knopfdruck den Ton des jeweiligen Videos wiedergeben, und der Anweisung: ")To watch the video, press the button and flick through the flipbook. Match the pace of the images to the audio.« (Fasshauer 2012), betonen in der Unmöglichkeit dieser Aufforderung nachzukommen, die Vermitteltheit der Ereignisse in Syrien. In der in Aussicht gestellten, aber unerreichbaren Re-Konfiguration des sezierten Bewegtbildes und der separierten Audiospur wird der Anschein der unmittelbaren Erfahrbarkeit auf eine medientechnologische Apparatur zurückgeführt. Appliziert auf Stempelkissen hinterlassen die Daumenkinos dabei blaue Farbe an den Fingern der Besuchenden und sprechen damit das Spannungsverhältnis zwischen der Unerfahrbarkeit und einer nichtsdestotrotz hergestellten Mitwissen- und Zeugenschaft an, die sich in der Interaktion mit diesen Videos vollzieht. Gleichzeitig erweitern sie das ästhetisch-diskursive Bedeutungsspektrum in zweifacher Weise: Nicht nur hinterlassen die Videos eine Spur bei den Betrachtenden, gehen also nicht spuren- oder folgenlos an ihnen vorüber. Auch die Betrachtenden selbst hinterlassen eine Spur, indem sie Tisch und Wand der Installation mit ihren blauen Fingerabdrücken markieren so wie sie einen digitalen Abdruck - ihre IP - bei dem Besuch einer Internetseite zurücklassen. Gleichzeitig werden kriminalistische und demokratische Diskurse durch die Ähnlichkeit zum Fingerabdruck in Verbrecher- und Wahlakten sowie zur Unterschrift auf Petitionen aufgerufen. Schließlich verweist ein von Hand abgespielter 8mm-Film des herangezoomten Blickkontakts zwischen Täter und Kamera/-mann in einer Endlosschleife auf die Amateurhaftigkeit der Handyvideoaufnahmen. Durch die enthaltene Referenz zum Super-8-Format ${ }^{19}$ steht diese der professionellen (Medien-)Gewalt des syrischen Regimes gegenüber, ohne dabei die bereits angesprochenen ästhetischen Bezüge zum Moment des Todes und zur Täterschaft zu verlieren. Zudem wird hier die kollektive Opferschaft des

und über sich hinausweisende Gewalt die Gerichtsverhandlung überschrieb. In diesem Sinn bringt auch die Videosektion Mroués nicht den Täter, sondern bloß das Phantom einer Gewaltvorstellung hervor, das dem syrischen Regime zugeschrieben wird.

19 Das von Kodak im Jahr 1965 eingeführte Super-8-Format revolutionierte das Amateurfilmen, indem es das Filmemachen massentauglich und u.a. das Genre der shome moviesı ermöglichte (vgl. Eastman Kodak 2016). 
syrischen Aufstandes und die Ausschnitthaftigkeit und Selektivität des Kamerabildes in dem Versuch thematisiert, den Filmenden über den Blickkontakt zum Täter in der Reflektion in dessen Augen sichtbarzumachen (vgl. Fasshauer 2012; Mroué 2012; Gespräch ${ }^{20}$ mit Mroué 2015: 2 u. 9).

Mroué präsentiert damit eine dekonstruktive Lesart der Handyvideos syrischer Aufständiger (vgl. Fasshauer 2012), die sich an Aspekten der Opfer-, Täter- und Zeugenschaft, dem Verhältnis von Bild und Tod sowie dem Verhältnis des Mediums Handykamera zum menschlichen Körper und zur (tödlichen) Gewalt entfaltet. Hierbei wird eine besondere Situiertheit des Handyvideos sichtbar, in der alternative Bedeutungen aus einem Aushandlungsprozess hervorgehen, an dem die Wissenssubjekte und Wissensobjekte gleichermaßen beteiligt sind. Denn wir als Betrachtende bleiben nicht außen vor, sondern werden Teil der Installation und damit in einem Wir der Opfer-, Täter- und Zeugenschaft, des Lebens und Sterbens im Syrienkonflikt eingeschlossen. Es ist ein bewegliches, niemals abgeschlossenes, performativ-hergestelltes Wir innerhalb eines figurativen Zusammenspiels ästhetischer und diskursiver Variablen. Hier werden nicht nur einem oder mehreren bestimmten auf YouTube verfügbaren Handyvideos eine Bedeutung zugeschrieben. Darüberhinaus erhalten der Tod, das Handyvideo und der Syrienkonflikt in einer je bestimmten Weise eine Bedeutung. Am Beispiel dieses einzelnen Handyvideos, das im Mittelpunkt der Kunstperformance und -installation von Mroué steht, auf YouTube in einem offenen Zuschreibungskontext verfügbar ist und von unterschiedlichen Medienkanälen in ihre eigenen Verwertungszusammenhänge gestellt wurde, offenbart sich damit ein Kaleidoskop an Bedeutungen. Je nach diskursiver Einbettung und ästhetischer Produktion und Rezeption sowie je nach Standort der Betrachtenden und ihrer Positionierung gegenüber dem Video stellt sich ein anderes Muster von Bedeutungen ein.

Dieses eine Handyvideo steht wiederum nicht alleine, sondern neben ihm, vor ihm und nach ihm, über und unter ihm steht ein Konglomerat an verwandten Videos aus dem Syrienkonflikt und anderen Konflikten wie dem Iran, dem Jemen, Ägypten oder Libyen, die ein komplexes Geflecht ganz unterschiedlicher Videoereignisse und Bedeutungszusammenhänge bilden. So finden sich auf YouTube neben Videos, die den Tod von Filmenden durch Waffengewalt aus dem Syrienkonflikt oder anderen Konflikten aus der Point-of-View-(POV)-Perspektive (`First-person<Todesvideos) zeigen, zahlreiche Videos, die den gewaltsamen Tod (noch im Prozess

20 Im Rahmen des vorliegenden Promotionsprojektes wurden Cespräche mit Kunstschaffenden, deren Werke zitiert werden, und mit Syrern, die direkt oder indirekt vom Syrienkonflikt betroffen sind, geführt. Mehr Informationen zu den geführten Gesprächen sind in Kapitel 2.4 zu finden. 
des Sterbens oder bereits vollendet) ${ }^{21}$ in der Beobachterperspektive (>Third-person`-Todesvideos) wiedergeben. Eine besondere Untergruppe stellen hier Enthauptungsvideos dar. Seit dem Todesvideo des US-amerikanischen Journalisten James Foley, das im August 2014 durch die jihadistische Organisation Islamischer Staat im Irak und in Syrien (ISIS) (heute nur noch als Islamischer Staat (IS) bezeichnet) veröffentlicht wurde, haben diese die öffentliche Wahrnehmung und internationale Debatte um den Syrienkonflikt und die damit in Zusammenhang stehenden Videos im Internet nachhaltig beeinflusst (vgl. Spiegel Online 2014a). Gleichzeitig trifft man ebenso auf investigative Videos, die bestimmte Todesvideos als Fälschungen $\mathrm{zu}$ entlarven und die damit in Verbindung stehenden >Fallgeschichten mit einem kriminalistischen Impetus aufzudecken suchen, wie auch auf offenkundige Fake-Todesvideos, die den Tod im Konfliktkontext parodieren. Eine spezielle Form der Enthüllungsvideos findet sich dabei in einer Reihe von Handyvideos, die sich als >Leaked-/Cell-phone-secrets $<-V i d e o s$ betiteln lassen und in der Art von geleakten oder enthüllenden Dokumenten Aufnahmen wiedergeben, die (vorgeblich) auf Handys von toten regierungstreuen Kämpfenden gefunden wurden und deren Brutalität zur Schau stellen sollen. Außerhalb von YouTube trifft man zudem auf unterschiedliche Reproduktionen von Handyvideos aus dem Syrienkonflikt: So werden Handyvideos nicht nur im Nachrichten- oder Kunstbereich zitiert (wie es bspw. bei dem beschriebenen netspanner-Video zu beobachten ist), sondern auch zum Gegenstand experimenteller und dokumentarischer Auseinandersetzungen im Filmbereich. Beispiele hierfür sind Birgit Heins >Found-footage<-Film ABSTRAKTER FILM (2013) (D, R: Hein) und Ossama Mohammeds und Siam Wimav Bedirxans Dokumentarfilm EAu Argentée - Syrie Autoportrait (2013) (F, R: Mohammed/Bedirxan).

Dieses lose Konglomerat an Videoereignissen und Bedeutungszusammenhängen scheint auf den ersten Blick in seinen uneindeutigen und mitunter gegenläufigen Erscheinungsweisen, die stets mit einem vermeintlich oder mutmaßlich versehen sein wollen, kaum fassbar zu sein. Bei genauerer Betrachtung verdichtet es sich jedoch zu einem engverflochtenen Phänomen, das sich als >Handy-Todesvideos bezeichnen lässt. Mit der begrifflichen Fokussierung auf das Handy bzw. Handyvideo soll dabei keineswegs die je eigene Spezifität anderer Medienapparate und -technologien und der mit ihnen verbundenen Praktiken, Diskurse und Ästhetiken

21 Der Begriff des Todes kann nach Schumacher (2004: 25ff.) in vier Bedeutungen ausdifferenziert werden: (1) die Sterblichkeit (als Beschreibung des Gesamtzusammenhanges von Leben und Tod); (2) das Sterben oder die Agonie, die normalerweise zum Ableben führt; (3) das Ableben oder das Im-Tode-sein (der Moment des Übergangs vom Leben zum Tod bzw. vom Sterben zum Zustand des Todes im Sinn einer Grenzüberschreitung); (4) der Zustand des Todes, der dem Ableben folgt (und die Bedeutung des Wortes Tod im eigentlichen Sinn als absoluter Gegensatz zum Leben benennt). 
negiert oder unterminiert werden. Doch obwohl sich nicht ausschließlich mit dem Handy aufgezeichnete Videos innerhalb des Phänomens versammeln, ${ }^{22}$ zeigt sich die mediale Apparatur des Handys bzw. das Handyvideo als diskursiver und ästhetischer Treiber in der Wahrnehmung und Bedeutung des Phänomens - spätestens seit dem Todesvideos der Neda Agha-Soltan im Iran 2009 (vgl. Meis 2013 u. 2014).

Für die Betrachtung in dieser Arbeit ist damit eine Begrifflichkeit entworfen, die von dem performativen Moment der betrachteten Videoereignisse ausgeht, d.h. dort ansetzt, wo ein Video als ein Handy-Todesvideo in Erscheinung tritt: wenn es an den Schnittpunkten Tod-Handyvideo-Syrienkonflikt mit anderen Videoereignissen zu dem übergeordneten Phänomen der Handy-Todesvideos konvergiert. Im Phänomen der Handy-Todesvideo stehen sich dabei alternative Bedeutungen des Todes, des Handyvideos und des Syrienkonflikts nicht etwa ausschließend gegenüber. Vielmehr überlagern sich unterschiedliche Bedeutungen in einem Modus des gleichzeitig Möglichen innerhalb eines steten Aushandlungsprozesses. Je nach Einbettung der Videos und ihrer Betrachtung kann die eine oder andere Bedeutung in der Wahrnehmung eines bestimmten Videoereignisses stärker hervortreten, ohne jedoch die alternativen Bedeutungen vollständig zu verdrängen. D.h. die ästhetische und diskursive Verfasstheit der Videos wie auch ihrer Erscheinungskontexte sind ebenso bedeutungsgewichtig wie der theoretisch-methodische Rahmen einer wissenschaftlichen oder der situative Rahmen einer nicht-wissenschaftlichen Betrachtung einschließlich der situierten Relation der jeweiligen Betrachtenden $\mathrm{zu}$ den Videos.

Wie in dieser Arbeit zu zeigen sein wird, steht das Phänomen der HandyTodesvideos dabei mit einer gleichzeitigen Ästhetisierung und Politisierung des Todes $^{23}$ in Zusammenhang, die gerade spezifische, miteinander existierende Be-

Ohne eindeutige Hinweise in der Beschreibung oder dem Titel der Videos kann häufig auch kaum zweifelsfrei bestimmt werden, ob ein Video tatsächlich mit einer Handykamera, einem Camcorder oder einer Helm-/Körperkamera aufgezeichnet wurde.

Eindrucksvolle Beispiele, in denen der Tod zur selben Zeit ästhetisiert und politisiert werden, liefern die künstlerischen Arbeiten von Teresa Margolles und die >Immersive-journalism «-Projekte von Nonny de la Peña. Die Mexikanerin Teresa Margolles arbeitet mit Leichen, Leichenteilen und verschiedenen Materialien des Bestattungsrituals von Personen ihres Lebensumfelds, die Opfer sozialer Missstände geworden sind: Drogen- und Bandenkriege, Prostitution und Frauenhandel, gesellschaftliche und polizeiliche Gewalt, militärischer und paramilitärischer Terror, Armut, Hunger und das Versagen des medizinischen Systems. Ihre Kunst soll die erschreckende Realität von Cewalt und Armut in Lateinamerika sowie die Toten dieser Realität sichtbar machen, ihnen eine Stimme zurückgeben und sich mit ihnen solidarisieren. (vgl. Macho 2007: 339ff.) Nonny de la Peña wiederum greift auf Amateurvideomaterial aus Nachrichtensendungen oder dem Internet zu aktuellen gesellschaftlichen Problemlagen, wie dem syrischen Bürgerkrieg und die syrische Flüchtlingskrise oder der herrschenden Ernährungsunsicherheit in den USA, zurück und übersetzt diese in eine virtuelle Realität mit Hilfe von Technologien, die auch bei Videospielen und Computersimulationen 
deutungen des Todes, des Syrienkonflikts und des Handyvideos samt seiner medientechnologischen Bedingtheit im Handy-Todesvideo hervorbringt. Der mit dem Begriff der Ästhetisierung ${ }^{24}$ adressierte Prozess, innerhalb dessen sich in Anlehnung an Hieber und Moebius (2011: 7ff.) eine Wahrnehmung und Beurteilung des Sozialen nicht allein zweckorientiert, sondern auch und zunehmend erlebnisorientiert und nach Aspekten des (eigenen) Gefallen vollzieht, bedeutet bezogen auf die Handy-Todesvideos eine Verschiebung der Wahrnehmung von der inhaltlichen Ebene hin zu der sinnlichen Ebene: von ihrem Abbildungs- und Repräsentationswert zu ihrem Erlebniswert und ihrer Fähigkeit, individuelle Wünsche und Ansprüche zu erfüllen. Gleichzeitig stehen dieselben Handy-Todesvideos gerade im Konfliktkontext mit einem Prozess der Politisierung in Zusammenhang, innerhalb dessen sie in den Widerstand gegen eine Gouvernementalität i. S. einer rationalregulierenden, politischen Souveränität eingebunden sind (vgl. Deuber-Mankowsky 2007: 165 u. 171f.). ${ }^{25}$ Der Aspekt der Politisierung des Todes soll mit Blick auf die Handy-Todesvideos entsprechend als eine widerständige Praxis adressiert werden, innerhalb derer in Videos thematisierte Tode in Bedeutungskämpfe eingebunden

Einsatz finden. Die Betrachtenden werden virtuell an den Ort des Ceschehens bzw. in das Ceschehen gebracht, um ihr Verständnis und ihre Bewusstwerdung für das, was dort vor sich geht und was es bedeutet gefährdet und in Not zu sein, zu erhöhen. (vgl. de la Peña et al. 2010; de la Peña 2014)

24 Der hier verwendete Begriff der Ästhetisierung rekurriert auf den Ästhetikbegriff in seiner ursprünglichen Bedeutung des griechischen Verbs saisthanomai< (ich nehme wahr) und des griechischen Nomens saisthetike` (Sinneswahrnehmung). Er bezieht sich somit nicht auf die (philosophische) Erforschung der Schönheit, ihrer Erkenntnis und/oder Beurteilung (vgl. Zettl 2001: 4; Scherke 2000: 110). Unter dem Oberbegriff der gesellschaftlichen Ästhetisierung wird dabei von verschiedenen Autoren die Ästhetisierung der Lebenswelt, des Sozialen oder der Cesellschaft verhandelt (vgl. u.a. Schulze 2000 [1992]; Scherke 2000; Hieber/Moebius 2011; Reckwitz 2012), der als gemeinsamer Nenner die Beobachtung einer (schub-, phasenweisen oder kontinuierlichen) Versinnlichung der Erfahrung zugrunde liegt. An deren Knotenpunkten werden häufig das Aufkommen, die Verbreitung und/oder Verstetigung von Medien oder mediatisierten Praktiken gesehen.

25 Nach Astrid Deuber-Mankowsky lässt sich Couvernementalität im Foucault'schen Sinn als eine Regierungspraxis beschreiben, die »zwischen den Polen der Rationalisierung [i. S. einer politischen Ökonomie; MM] und der politischen Souveränität angesiedelt ist und beide in gewisser Weise verbindet. « (2007: 154) Nach Foucaults Konzeption ist die Gouvernementalität eng mit dem Begriff der Biopolitik, d.h. mit bevölkerungsoptimierenden und realitätskonstituierenden Maßnahmen und Regulationen eines Regierungshandeln, verknüpft (vgl. ebd.: 155 u. 152). In Abgrenzung zu einem politikwissenschaftlichen Verständnis wird in dieser Arbeit mit dem Begriff der Politisierung damit nicht ein Prozess fokussiert, in dem gesellschaftliche Problemlagen, Themen oder Sachverhalte Gegenstand einer öffentlichen Entscheidungsfindung und der Aushandlung des öffentlichen Interesses werden (vgl. ebd.: 153; bpb 2012; Zürn 2013: 13). 
sind, in denen sich eine regierende Macht und eine (oder mehrere) dieser regierenden Macht unterworfene(n) oder antagonistisch gegenüberstehende(n) Gruppe(n) von Menschen (wie eine Zivilbevölkerung, oppositionelle militärische, paramilitärische oder jihadistische Akteure) gegenübertreten. Diese Einbindung kann sowohl in individualisierter als auch generalisierter Form durch die Verknüpfung mit sozialen Problemlagen, Missständen u.ä., die Hintergrund eines Konflikts sind, erfolgen.

Im Phänomen der Handy-Todesvideos zeigt sich entsprechend dieser gleichzeitigen Ästhetisierung und Politisierung des Todes eine oszillierende Verschiebung der mit dem Tod allgemein und den Toden im Syrienkonflikt speziell verbundenen gesellschaftlichen Diskurse der Gewalt und Trauer, des Martyriums und Heldentums, der Evidentialität und Justiziabilität, der Pornografie und Obszönität, des Terrorismus und Extremismus, der Erlebnisorientierung und Spektakularisierung zwischen den Sphären des Ästhetischen und des Politischen. Entsprechend darf sich diese Arbeit nicht auf einzelne Aspekte verengen - was der Komplexität des Phänomens kaum gerecht werden würde -, sondern muss eine möglichst weite Betrachtung des ästhetischen und diskursiven Spektrums des Phänomens der Handy-Todesvideos anstreben. Eine solche Betrachtung stellt sich angesichts der Heterogenität des Phänomens als ein komplexes Unterfangen dar, das nach einer relationalen Betrachtungs- und Beschreibungsweise verlangt. Denn wie diese Einführung gezeigt hat, vereint das Phänomen ästhetisch wie auch diskursiv nicht nur ganz unterschiedliche Videoereignisse in sich. Auch ist dem Phänomen eine spezifische Situiertheit inhärent, die zum einen den Tod, das Handyvideo und den Syrienkonflikt sowohl darin verankert als auch über diese hinausweist: Als mitkonstituierend tritt nicht nur der Syrienkonflikt in Erscheinung, sondern auch mit ihm gesellschaftspolitisch und ikonografisch verwandte Konflikte, die ähnliche Videooder Bildereignisse und damit verbundene Praktiken hervorbringen. Nicht nur ist der Tod als singulärer Augenblick dem Phänomen eingeschrieben, sondern auch gewaltsame Handlungen, die ein Überleben oder Sterben zur Folge haben (können). Nicht nur mit den Handyvideos materialisiert sich das Phänomen, sondern auch mit medienästhetisch und -historisch verwandten fotografischen, filmischen wie auch videobasierten Medien gemeinsam mit ihren unterschiedlichen Erscheinungskontexten, die von sozialen Medien, über Filmfestivals und Kunstausstellungen zu Printmedien reichen können. Zum anderen sind die Betrachtenden selbst in ihrer Situiertheit wie auch kollektiv als Zuschauende, Kommentierende, weiterverbreitende und -verarbeitende sowie direkt oder indirekt Konfliktbetroffene Mitkonstituierende des Phänomens sowie durch das Phänomen Mitkonstituierte. Das Phänomen der Handy-Todesvideos ist damit weder in seinen Erscheinungen noch in seinen Bedeutungen festschreibbar. Nachdem die Arbeit nun im Folgenden wissenschaftlich verortet wird, wird die Fragestellung und das Phänomen der Handy-Todesvideos in seiner Situiertheit und Verbundenheit mit einer Ästhetisie- 
rung und Politisierung des Todes genauer umrissen. Daraus hervorgehend wird eine im zweiten Kapitel noch auszuführende diffraktive Forschungsperspektive begründet, die die Betrachtung des Phänomens der Handy-Todesvideos leiten wird.

\subsection{Das Phänomen der Handy-Todesvideos und die Ästhetisierung und Politisierung des Todes}

Angesichts der bisherigen wissenschaftlichen Beschäftigung mit dem Syrienkonflikt und dem Einsatz sozialer Medien im Konfliktkontext weist die Betrachtung des Phänomens der Handy-Todesvideos diese in eine neue Richtung. In kommunikations- und sozialwissenschaftlichen Auseinandersetzungen (insb. politikwissenschaftlicher Fokussierung) mit der Nutzung sozialer Medien (speziell der Veröffentlichung und Verbreitung von Amateurvideos auf Internetplattformen) im syrischen Bürgerkrieg treten diese als zentrale, wenn nicht gar revolutionierende Kraft der Repräsentier- und Erfassbarkeit des Konflikts wie auch der Konfliktführung auf. Der Syrienkonflikt selbst wird hierbei als Initiator neuartiger Einsatzmöglichkeiten sozialer Medien und des Internets durch politische Akteure, aufständische und terroristische Gruppen und Journalisten gesehen. Diese würden mit alternativen Kommunikationsstrategien, Handlungsmustern und Gruppendynamiken sowie einer Veränderung der Beziehung zwischen neuen und traditionellen Medien einhergehen und werden als prägend für zukünftige Konflikte eingeschätzt (vgl. u.a. Seo/Ebrahim 2016; Lynch/Freelon/Aday 2014; Ali/Fahmy 2013; Khamis/Gold/Vaughn 2012; Dauber 2009).

Die von der Friedensforschung und Konfliktprävention informierte Studie Syria's Socially Mediated Civil War (2014) von Marc Lynch, Deen Freelon und Sean Aday zeigt insbesondere die wesentliche Funktion kurativer Knoten in sozialen Netzwerken auf. Diese nähmen eine ebenso machtvolle Rolle in der öffentlichen Sichtbarwerdung von Fotos, Videos und Themen ein wie Fernsehproduzenten oder Zeitungsherausgeber. Die Autoren heben die Notwendigkeit eines differenzierteren Verständnisses der strukturellen Verzerrung (engl. >structual bias`) in sozialen Medien und der Herausforderungen an einen kurativen Aktivismus hervor. Ebenso betonen sie die erforderliche Entwicklung besserer Methoden der Sentimentanalyse. ${ }^{26}$ Diese müssten über die Authentifizierung bestimmter Videos und die Überprüfung spezifischer Behauptungen hinausgehen, um zu gesicherten Aussagen der politischen Bedeutung von identifizierten Clustern und Trends zu gelangen (vgl. 
ebd.: 26f.). Dabei lassen die Autoren allerdings die Effekte für eine ebensolche Bedeutung unberücksichtigt, die aus den spezifischen ästhetischen Verfasstheiten, medialen Erscheinungskontexten und damit verbundenen diskursiven Einbettungen von videobasierten Aufnahmen gewaltsamer Auseinandersetzungen und anderer kriegerischer Handlungen hervorgehen, indem diese allgemein unter dem Sammelbegriff der sozialen Medien gefasst werden. Die Einbeziehung solcher ästhetischen und diskursiven Partikularitäten verschiebt jedoch, wie diese Arbeit herauszustellen sucht, den Blick der identifizierten Cluster und Trends und öffnet ihn für alternative, überlagernde Bedeutungen, die andernfalls außerhalb des wissenschaftlichen Fokus bleiben.

Aus einer militärwissenschaftlichen Perspektive befasst sich Cori E. Dauber in ihrer Studie YouTube War: Fighting in a World of Cameras in Every Cell Phone and Photoshop on Every Computer (2009) allgemeiner mit Video- und Onlinepraktiken terroristischer Organisationen (bspw. al-Qaida und die Taliban) im Zuge des Irakkrieges (2003-2011) nach 9/11 sowie mit dem Umgang der traditionellen Nachrichtenmedien mit diesen. Sie stellt nicht nur eine Kategorisierung und segmentierte Analyse unterschiedlicher terroristischer Videos zur Verfügung. Sie befasst sich insbesondere auch mit der Ikonografie von Enthauptungs- und Tötungsvideos und deren öffentlichen Wiedergabe in den westlichen Nachrichtenmedien sowie deren Einbettung in die politische Debatte (vgl. ebd.: 62ff.). Dabei betrachtet Dauber die Videos aus einer kriegsstrategischen Zentrierung heraus als ausgeklügelte terroristisch motivierte Medienevents, die eine neue Form der Kriegsführung mit sich brächten. In ihrer daran anknüpfenden Einschätzung der öffentlichen Resonanz auf die fragmentierte (sprich: zensierte) Wiedergabe von Gewalt- und Tötungsvideos auf Nachrichtenkanälen, reduziert sie den Erscheinungskontext von Onlinevideoplattformen wie YouTube auf ein Sammelbecken für nicht-reglementiertes Videomaterial. Diese würden eine verfälschte Realität und Unwahrheiten verbreiten, dem eine akkurate Version von Ereignissen und Sachverhalten gegenübergestellt werden müsse (vgl. ebd.: 85ff.). Jedoch ist es gerade die Hervorbringung und das $\mathrm{Ne}-$ beneinanderexistieren alternativer Realitäts- und Wahrheitsansprüche, die diese Onlinevideos gemeinsam mit ihren Re-Produktionen und Re-Materialisierungen in anderen medialen Erscheinungskontexten und Formaten zu einer bedeutenden Zwischen- und Endstation in der Rezeption von Kriegen und Konflikten werden lässt. Hier liegt die besondere Herausforderung, der diese Arbeit begegnen möchte, in der Frage nach der Wahrnehmung und Bedeutung dieser Videos im Kriegsund Konfliktkontext.

Kari Andén-Papadopoulos' kommunikations- und medienwissenschaftliche Arbeit Body Horror on the Internet: US Soldiers Recording the War in Iraq and Afghanistan (2009) zur Produktion und Verbreitung extremgewaltvollen Fotomaterials und ebenso gewaltvoller Kommentare am Beispiel des >online bulletin boards 
NowThat'sFuckedUp.com (NTFU) ${ }^{27}$ befasst sich mit den Bedeutungen und kommunikativen Funktionen von gewaltvollem Bildmaterial, das von Soldaten im Einsatz im Irak und in Afghanistan online gestellt wurde. Hierbei fokussiert Andén-Papadopoulos die individuelle Motivation hinter der Produktion und Verbreitung eines solchen Bildmaterials als psychologisches Symptom im Sinn eines affektiven Wiederdurchlebens einer traumatischen Kriegserfahrung. Damit verengt sie jedoch die Bedeutung und kommunikative Funktion gewaltvollen Bildmaterials im Kriegs- und Konfliktkontext auf den partikularen Bereich einer selbsttherapeutischen Maßnahme und klammert die Bedeutung und kommunikative Funktion reproduzierender und -publizierender Praktiken und Aneignungen aus. Gerade dies ist jedoch ein Moment, wie bereits im vorherigen Abschnitt hervorgehoben wurde, der mit der Verortung dieses Bildmaterials in der Onlineumgebung sozialer Medien nicht ignoriert werden sollte. Auch droht hier der Blick für alternierende und gleichzeitige Bedeutungen und Wahrnehmungen in der Betrachtung verstellt zu werden, wenn Andén-Papadopoulos'Ansatz von der Vorstellung einer zugleich realitätsauthentifizierenden und -auslöschenden Funktion geleitet ist. Um diesem Diserderat zu begegnen, strebt diese Arbeit gerade einen nicht verengenden Blick an, der die Alternation und Gleichzeitigkeit von Bedeutungen und Wahrnehmungen in der Betrachtung von gewaltvollem Bildmaterial im Kriegs- und Konfliktkontext zulässt.

Die Arabistin und Politologin Cécile Boëx beschäftigt sich wiederum in ihrer Auseinandersetzung YouTube and the Syrian Revolution. On the Impact of Video Recording on Social Protests (2017) mit dem Effekt der Videoaufnahme als Praktik in sozialen Protesten. Sie betrachtet hierbei den Verlauf der Revolte aus der Perspektive der Evolution der videografischen Praxis und der audiovisuellen Grammatik, die sie hervorbringt. Boëx setzt hierbei explizit an den Videos als eine neue Form der medialen Kommunikation an, um zu betrachten, wie diese Praktiken, Diskurse und

27 NTFU war ein von Frühjahr 2004 bis Frühjahr 2006 aktives, privates Onlineforum, das als Webseite für Amateurpornographie startete und unter US-Soldaten stationiert in Übersee populär wurde. Diese hatten jedoch Schwierigkeiten die Mitgliedschaftsgebühren zahlen, wenn sie in Irak oder Afghanistan stationiert waren, da die Kreditkartenunternehmen Belastungen aus als starkgefährdet eingestuften Cebieten blockierten. Die Webseitenbetreiber führten daraufhin die Regelung ein, dass US-Truppen ein gebührenfreier Zugriff gewährt wurde, wenn sie authentische Bilder auf die Webseite hochluden, die ihre Stationierung in Übersee belegten. In diesem Zuge wurden nicht nur Aufnahmen von Landschaften, Sandstürmen und Selbstportraits vor schwerer Artillerie gepostet, sondern auch Nahaufnahmen von verstümmelten Körpern toter irakischer Aufständischer und Zivilisten. Diese Aufnahmen lösten einen Hype auf NTFU aus und im September 2005 machte die Webseite unter dem Stichwort »trading gore for porn« (ebd.: 926) internationale Schlagzeilen. 
Vorstellungen formen. ${ }^{28}$ In ihrer Fokussierung auf die visuelle Dramaturgie der Videobilder und der Strategie des filmenden Handelns innerhalb einer Protestpraxis identifiziert Boëx (2017) zwar die Bedeutung der Videos als eine Praktik der Herausforderung der machthabenden Autoritäten und der Zurückforderung des öffentlichen Raums der Straße in der Frühphase des Syrienkonflikts. Auch stellt sie diese als eine Praktik der Sichtbarmachung und öffentlichen Identifikation des Protests in der späteren Phase des Syrienkonflikts heraus. Sie diagnostiziert den Videos hierbei allerdings eine geringe politische Wirksamkeit, als dass die Mehrzahl der Videos keine große Anzahl an sviews erreicht habe und allgemein nicht in der Lage gewesen sei, die Kräfterelationen in der Region zu beeinflussen. (vgl. ebd.: 13) Unberücksichtigt bleiben bei Boëx auch die Republikationen und Reproduktionen von Videos in anderen Erscheinungskontexten, wie auch ihre Rematerialisierungen in anderen medialen Formaten. In einzelnen Fällen haben solche Republikationen, -produktionen und -materialisierungen einen starken diskursiven und ästhetischen Widerhall erfahren (wie bspw. das eingangs zitierte netspanner-Video). Als singuläre Ereignisse wie auch als eingebettetes Ereignis in ein umfassenderes Konglomerat an videobasierten Materialisierungen haben diese den Diskurs und die Ästhetik des Syrienkonflikts und der Videos nachhaltig beeinflusst. Auch tragen sie, wie diese Arbeit im vierten Kapitel zeigen wird, eine signifikante politische Bedeutsamkeit in sich, die sich nicht auf eine Veränderung der politischen Lage im Syrienkonflikt selbst reduzieren lässt, sondern die Wahrnehmung von direkt und indirekt Konfliktbetroffenen und politisch ausgerichtete Reaktionen von Akteuren abseits des Syrienkonflikts einschließt.

Die Vielzahl und Diversität an verfügbaren Videoaufnahmen des Todes im Syrienkonflikt auf sozialen Onlineplattformen in sogenannten >rohen arbeiteten Versionen ebenso wie deren Reproduktion in anderen Erscheinungskontexten (u.a. im journalistischen, künstlerischen und filmischen Bereich), verweist dabei auf die Relevanz einer wissenschaftlichen Auseinandersetzung mit den Bildern des Todes. Richtungsweisend ist hier die Bild-Anthropologie des Kunsthistorikers und Medientheoretikers Hans Belting (2001), innerhalb derer die Bilder des Todes als Kulturtechnik in den Blick kommen. Belting (ebd.: 11f.) führt hierin einen Bildbegriff ein, der sowohl die räußeren d.h. getragen durch ein Medium und seine bildgebenden Verfahren, als auch die >inneren Bilder der menschlichen Imagination umfasst, die gemeinsam die symbolische Einheit >Bild k konstituieren. Diesen Bildbegriff setzt er in seiner Auseinandersetzung mit dem Verhältnis von Bild und Tod über verschiedene sozio- und medienhistorische Epochen und Kulturen ein. Mit diesem offenen Bildbegriff gelingt es ihm, die sich wandelnden bildlichen Repräsentationen der Toten und des Todes 
und eine dahinterliegende soziokulturelle Bild- und Todespraxis in die Betrachtung einzubeziehen. Gleichzeitig kann er eine sich wandelnde Todeserfahrung am Bild und eine Bilderfahrung am Tod damit in Beziehung setzen. Übertragen auf das Phänomen der Handy-Todesvideos lassen sich damit eine Vorstellung des Todes im Syrienkonflikt und die medialen Materialisierungen des Todes im Handyvideo als gemeinsame Hervorbringende von ineinandergreifenden soziopolitischen Bedeutungen des Todes im Syrienkonflikt verstehen.

Hieran anschließend ist auch der kunst- und kulturwissenschaftlich orientierte Band Die neue Sichtbarkeit des Todes herausgegeben von Thomas Macho und Kristin Marek (2007) zentral. Der Tod wird hier im Spannungsverhältnis der Un-/Möglichkeiten seiner Bildwerdung zum Knotenpunkt ästhetischer und diskursiver Praktiken des Einschlusses und Ausschlusses und der Aushandlung von sozialen Un/Sichtbarkeiten, die mit den neuen Medien (hierzu werden die Fotografie, der Film und digitale Medien gezählt) einem vermeintlichen Wandel unterliegen (vgl. Macho/Marek 2007: 9ff.). Die aktuellere Arbeit Dying in Full Detail (2017) der Filmund Medienwissenschaftlerin Jennifer Malkowski wiederum konzentriert sich ausschließlich auf die medialen und sozialen Erscheinungsmöglichkeiten des Todes in Film und Video. Vor dem Hintergrund des Aufkommens digitaler Technologien nimmt sie die damit verbundenen ethisch politischen Implikationen in den Blick. Digitale Medientechnologien erscheinen hierbei als quantitative Kapazitätssteigerer des Aufnehmens und Verbreitens des Anblicks des Todes, welche die Politisierung individueller Tode durch die rhetorische Kraft ihrer dokumentarischen Repräsentation begünstigen (vgl. Malkowski 2017: 6f.). Die soziologische Auseinandersetzung Sterben als Spektakel -Zur kommunikativen Dimension des politisch motivierten Suizids von Lorenz Graitle (2011) behandelt das aktuelle Phänomen der Selbstmordanschläge und die damit verbundene mediale Inszenierung. Einbezogen werden in die Analyse neben Abschiedsbriefen in Papier- und digitaler Form videobasierte >Märtyrer-Testamente<. Letztere spielen auch im Rahmen des Phänomens der Handy-Todesvideos in der Materialisierung als Märtyrervideos eine signifikante Rolle (vgl. Kapitel 3.2). Einer kommunikations- und sozialwissenschaftlichen Tradition folgend, stützt sich Graitle (2011: 129ff.) in seiner detaillierten Betrachtung von sieben Abschiedsdokumenten auf eine rein textliche und inhaltsbasierte Analyse, die die spezifischen Effekte der verschiedenen medialen Erscheinungskontexte (Veröffentlichungen in Zeitungen, Fernsehnachrichten oder auf Internetseiten) unterthematisiert lässt. Ebenso unberücksichtigt bleibt die unterschiedliche ästhetische Qualität der medialen Erscheinungsform, die Rückschlüsse auf die kommunikative Wirksamkeit politisch motivierter Selbstmorde zulässt.

Je nach Schwerpunktsetzung werden in den zitierten wissenschaftlichen Arbeiten der Tod und seine medialen Materialisierungen als übergreifende Ereignisse diskutiert, denen eine transzendierende Bedeutung innewohnt. Als situierte Ereignisse in bestimmten Erscheinungskontexten, die innerhalb eines umfassenderen 
Phänomens zusammen und in ihren Bedeutungen als voneinander abhängig zu denken sind, werden sie nicht begriffen. Entsprechend ist es angesichts des Phänomens der Handy-Todesvideos sinnvoll, nach der Bedeutung bzw. nach alternativen Bedeutungen des Todes, des Handyvideos und des Syrienkonflikts im HandyTodesvideo zu fragen. Betrachtet werden muss, wie diese Bedeutungen aufeinander angewiesen sind, miteinander existieren, sich überlagern und das Phänomen selbst hervorbringen:

Was bedeutet der Tod, das Handyvideo und der Syrienkonflikt im Handy-Todesvideo? Wie wirken alternative Bedeutungen des Todes, des Handyvideos und des Syrienkonflikts ineinander und bringen das Phänomen der Handy-Todesvideos hervor?

Hierbei soll eine Forschungsperspektive verfolgt werden, die ästhetische und diskursive Aspekte mit dem Ansatz des situierten Wissens in Einklang zu bringen sucht. Unter den ästhetischen Aspekten sind sowohl die medientechnologischen Bedingtheiten als auch die medienspezifische Materialität der Handy-Todesvideos in einer Onlineumgebung sozialer Medien mit zu adressieren. Hinsichtlich der mit den Handy-Todesvideos verbundenen diskursiven Effekte sind gerade auch medienhistorische und ikonografische Bezüge zentral, wenn einerseits die Rematerialisierungen von Handy-Todesvideos und andererseits gesellschaftshistorische Motive mit religiösen, politischen und/oder kulturellen Bezugspunkten in den Blick kommen.

Die Wechselwirkung von ästhetischen und diskursiven Bedeutungseffekten ist dabei ein Gesichtspunkt, der in der wissenschaftlichen Auseinandersetzung mit der Rolle von Medien in Kriegen und Konflikten häufig unterberücksichtigt bleibt. Denn, obwohl der kanadische Medientheoretiker Marshall McLuhan mit seinem populären Statement »the medium is the message« (1964: 7) auf die Bedeutsamkeit der ästhetischen Ebene der Medien vor nun mehr als einem halben Jahrhundert hingewiesen hat, gehen gerade sozial- und kommunikationswissenschaftliche Analysen häufig auch heute nicht über die inhaltliche Ebene hinaus (vgl. Zettl 2011: 11). Exemplarisch zeigt sich dies bei Ben O'Loughlin (2011: 85f.), der zwar vor dem Hintergrund der Ergebnisse der >Shifting-Securities -Studie auf ästhetische Effekte - ohne diese allerdings explizit als solche zu benennen - in der Wahrnehmung von Videomaterial US-amerikanischer Luftangriffe im Irak 2003 verweist. Diese bringt er jedoch nicht weiter mit der öffentlichen Resonanz in Verbindung. Die shifting-Securities<-Studie ist dabei selbst ein Beispiel für die Ausklammerung ästhetischer Aspekte in der wissenschaftlichen Auseinandersetzung mit einem medienbezogenen Phänomen, wenn in der multidisziplinären Betrachtung der Hervorbringung und Veränderung von Sicherheits- und Konfliktdiskursen im Vereinigten Königreich nach dem Irak-Krieg 2003 eine Zuschauerethnografie und Experteninterviews mit einer lediglich textlichen Analyse von Fernseh- und Internetnachrichten ergänzt wird (vgl. ebd.: 75; Gillespi 2007: 26ff.). Ein neueres Beispiel für eine sozial-/kommunikationswissenschaftlich ausgerichtete Medienanalyse, in der 
ästhetische Aspekte vernachlässigt werden, ist die vergleichende Framing-Analyse zur visuellen Propaganda auf Facebook im Syrienkonflikt von Hyunjin Seo und $\mathrm{Hu}-$ sain Ebrahim (2016: 228 u. 233). Hier wird eine inhaltliche und strukturell formale Bildanalyse mit einer numerischen Auswertung der Reaktionen von Nutzenden anhand der Anzahl der Likes, Shares und Kommentare kombiniert.

Die hier zu entwerfende Forschungsperspektive muss in Hinblick auf das Phänomen der Handy-Todesvideos nicht nur notwendig auf einen wechselseitigen Bezug zwischen diskursiven und ästhetischen Variablen der zu betrachtenden zentralen medialen Materialisierung - dem Handyvideo - aufbauen. Wesentlich ist auch, den Tod selbst weder auf ein ästhetisches Ereignis zu reduzieren noch seine ästhetische Dimension unterberücksichtigt zu lassen. Denn im Phänomen der HandyTodesvideos stehen, wie im vorangegangenen Abschnitt bereits angedeutet wurde, eine Ästhetisierung und Politisierung des Todes in einem eng verflochtenen Verweisungsverhältnis zueinander. Dieses lässt sich prägnant an der sich wandelnden ästhetischen und diskursiven Verfasstheit der Handy-Todesvideos aus dem Syrienkonflikt auf YouTube ablesen: Während in den ersten beiden Jahren des Konflikts noch eine große Zahl der hochgeladenen Videos von geringerer Bild- und Tonqualität waren, weisen Videos, die seit 2013 hochgeladen wurden, eine zunehmend höhere Bild- und Tonqualität auf, ohne dabei ihre weiteren >Low-tech--Spezifika (wie Synchronton, unmittelbarer Blickwinkel, unscharfer Fokus, zu schnelle Schwenks, schräge Kameraperspektive und reduziertes oder nicht-vorhandenes >editing (vgl. Krenzer 2013: 43; Fiske 2002: 389)) zu verlieren. Dabei >werben $<$ diese Videos geradezu mit einer unmittelbaren Kriegserfahrung in HD- und GoPro-Optik ${ }^{29}$ und wecken eher die Assoziation eines filmischen, videospielerischen oder pornografischen Erlebnisses als eines authentischen Miterlebens von tatsächlichen Straßenkämpfen in Syrien (vgl. Krautkrämer 2014a: 124). Wenn hierbei in der anfänglichen Protestphase bis ca. Mitte 2011 die Videoaufnahmen vor allem durch das Bedürfnis ein außergewöhnliches, revolutionäres Ereignis festzuhalten motiviert waren, änderte sich dies in dem Moment als das syrische Regime mit militärischer Gewalt gegen die Proteste vorging und aus friedvollen Straßenprotesten mehr und mehr brutale Auseinandersetzungen wurden. Zunehmend zeichnete sich eine politische Motivation hinter der Aufnahme von Videos (oder deren Verhinderung) ab, in der es nicht bloß darum ging, eine moralische Entrüstung in Bilder und Worte zu fassen. Vielmehr stand im Vordergrund die anhaltende militärische Gewalt und Unterdrückung wie auch Kriegsverbrechen und Menschenrechtsverletzungen als (potentiell juristische) Beweisstücke zu dokumentieren oder ebendiese $\mathrm{zu}$ dementieren und

29 Vgl. u.a. WarLeaks (2014) <https://www.youtube.com/watch?v=tfEjTgUmeWw>, squidd81 (2016) <https://www.youtube.com/watch?v=atRahVIINeo> und R\&U Videos (2018) <https:// www.youtube.com/watch?v=Wjh5iKJ16Lg>. 
eliminieren. Zur selben Zeit erschienen auch Videoaufnahmen von unterschiedlichen Konfliktgruppen, die einschüchternd oder diskreditierend motiviert waren. (vgl. Wessels 2016: 33f.) Mit der steigenden Aufnahmequalität ab 2013 zeigt sich wiederum auch eine Verschiebung in der hinter den Videos liegenden Motivation, wenn vor allem die Aufzeichnung eines außergewöhnlichen Kriegserlebnisses die Filmenden anzutreiben scheint - insbesondere dann, wenn es sich um unbearbeitetes Material in Längen von bis zu 30 Minuten handelt (vgl. ebd. 44).

Diese Beobachtung auf eine Ästhetisierung und Politisierung des Todes engführend, lässt sich das Phänomen der Handy-Todesvideos, wie diese Arbeit zeigen wird, damit als Konsequenz einer ambivalenten, aber enggewebten videografischen Praxis im Syrienkonflikt beschreiben: Einerseits werden die Todesvideos zunehmend in den Bedeutungskontext einer mehr oder weniger losen Community oder Interessensgemeinschaft gestellt, in dem sie mitunter abseits von einer repräsentierenden und abbildenden Funktion rein nach ästhetischen Gesichtspunkten im Rahmen von Unterhaltungs- oder Freizeitaktivitäten wahrgenommen, beurteilt und gestaltet werden. Andererseits werden dieselben Videos zur gleichen Zeit in denselben Veröffentlichungs- und Distributionskontexten (wie einem YouTubeKanal) zum Gegenstand von Meinungsbildungs-, Aufklärungs-, Solidarisierungs-, Mobilisierungs-, Selbstvermarktungs- oder Propagandastrategien. Diese Strategien beschränken sich dabei nicht auf den Erscheinungskontext sozialer Medien. Ebenso erstrecken sie sich über die Erscheinungskontexte der Nachrichtenmedien, der Kunst, des Kinos bzw. Films und der virtuellen Realität. Dort verlieren sie nicht etwa ihr politisches Potential, sondern weiten es beständig aus.

Eine Trennung der Aspekte der Ästhetisierung und Politisierung des Todes in der wissenschaftlichen Betrachtung des Phänomens der Handy-Todesvideos würde eben diese Gleichzeitigkeit und dieses Ineinanderwirken von ästhetischen und politischen Motivationen und Effekten unbeachtet lassen, die das Phänomen auszeichnen. Eine Reduktion des Todes auf ein rein ästhetisiertes Phänomen würde insbesondere seine existentielle Dimension, die gerade auf den Ort und den Moment seines Auftretens verweist, ausklammern. Damit würde eine Betrachtung der soziopolitischen Bedeutsamkeit der diskursiven Sichtbarkeit des Todes droht verstellt zu werden, wenn diese Sichtbarkeit in den Bereich eines - mitunter als verwerflich markierten - Spektakels verrückt wird (vgl. Kapitel 4.1.2). Den Tod wiederum als rein politisiertes Phänomen zu diskutieren läuft Gefahr gerade solche Momente einer sensationsmotivierten Wahrnehmung zu überschreiben, die ein wesentlicher Treiber seiner Sichtbarkeit im Sinn der Veröffentlichung, Verbreitung und Weiterverarbeitung von Bildmaterial vor allem in der Onlineumgebung sozialer Medien ist. Eine Ausklammerung der ästhetisierten Dimension des Todes würde zur selben Zeit seiner gesellschaftlichen Tabuisierung Vorschub leisten, die die Betrachtung des Todes bzw. seiner Bilder als Obszönität brandmarkt und in letzter Instanz den Tod selbst außerhalb des Diskurses, d.h. abseits des gesellschaftlichen 
Sicht- und Sagbaren, verortet. Dieses Moment ist insbesondere bezogen auf Tode im Konfliktkontext sowie der mit ihm zusammenhängenden Frage der Betrauerbarkeit selbst häufig politisch motiviert (vgl. Butler 2004: 34f.; s.a. Kapitel 3.1.1 u. 4.1).

Wie im vorangegangenen Abschnitt dargestellt, darf auch in der wissenschaftlichen Betrachtung und Beschreibung nicht außer Acht gelassen werden, dass die Sichtbarkeit und das In-Erscheinung-treten der Handy-Todesvideos stets Ausdruck sowie Hervorbringungsmodus der Situiertheit der Betrachtenden wie auch des betrachteten Phänomens selbst ist. Für eine medienkulturwissenschaftliche Betrachtung und Beschreibung des Phänomens der Handy-Todesvideos stellt sich somit die Frage: Wie kann das Phänomen der Handy-Todesvideos in seiner Situiertheit und Komplexität an Ereignissen und Bedeutungen sowie in seiner Verbundenheit mit einer Ästhetisierung und Politisierung des Todes methodisch sinnvoll betrachtet und beschrieben werden?

Wie im folgenden Kapitel zu zeigen ist, wird hierfür eine Betrachtungs- und Beschreibungsweise benötigt, die in der Lage ist, diskursive und ästhetische Effekte, die kontext- und betrachtungsbezogenen Bedingtheiten der Videos wie auch die Effekte der Ästhetisierung und Politisierung, die aus den Auseinandersetzungen der Nutzenden, Konfliktbetroffenen und -beteiligten, Kunstschaffenden, Filmemachenden, Medienschaffenden und Forschenden mit den Handy-Todesvideos resultieren, gleichermaßen in der Hervorbringung des Phänomens zu berücksichtigen - ohne dabei in die Festgeschriebenheit einer Bedeutung der HandyTodesvideos zu verfallen, ohne eine Bedeutung über eine andere oder alle anderen zu stellen, ohne divergente Bedeutungen als sich ausschließende Gegensätze eines antagonistischen Deutungskampfes zu verstehen, sondern als produktive Momente des gleichzeitig Möglichen zu erfassen. Ziel muss es sein, eine Geschichte der Handy-Todesvideos zu schreiben, in der unterschiedliche und zuweilen divergente Bedeutungen des Phänomens nicht bloß nebeneinanderstehen, sondern sich gegeneinander verschieben und überlagern, Verbindungen eingehen und neue, andere Bedeutungen hervorbringen. Diese Geschichte darf dabei nicht in einer retrospektiven Analyse und nachfolgenden Synthese eines rekonstruktiv gedachten Sinnzusammenhangs münden. Sie muss gerade das generative und fragile Moment der Bedeutung sowie die Situiertheit der Handy-Todesvideos herausstellen. Dies soll in einer Betrachtungs- und Beschreibungsweise umgesetzt werden, die die Gegenwartsbezogenheit und -bedeutsamkeit der Betrachtung des Phänomens fokussiert und dabei seine Zukunfts- und Vergangenheitsgerichtetheit, d.h. seine Unabgeschlossenheit und Instabilität, im Blick behält. Der hierfür im nächsten Kapitel präsentierte Vorschlag gründet sich auf das optische Bild der Diffraktion, welches Donna J. Haraway in ihren Arbeiten als Ausgangspunkt für eine perspektivische Neuausrichtung des wissenschaftlichen Betrachtens und Schreibens gedient hat. 
